\title{
DESIGN AND SYNTHESIS OF 4-SUBSTITUTED QUINAZOLINE DERIVATIVES FOR THEIR ANTICONVULSANT AND CNS DEPRESSANT ACTIVITIES
}

\author{
BISWAJIT DASH ${ }^{1}$, SUVAKANTA DASH ${ }^{1}$, DAMIKI LALO0 ${ }^{1}$ \\ ${ }^{1}$ Girijananda Chowdhury Institute of Pharmaceutical Science, Azara, Hathkhowapara, Guwahati 781017, Assam \\ Email: biswajit.itbhu@gmail.com
}

Received: 01 Oct 2016 Revised and Accepted: 17 Nov 2016

\begin{abstract}
Objective: The present work is designed to synthesise some isomeric new series of Quinazoline-4-one/4-thione derivatives, based on the pharmacophoric model of central nervous system (CNS) activity by structural modifications retaining the essential structural features for the activity and evaluated for their anticonvulsant and CNS depressant properties.
\end{abstract}

Methods: A series of 7-chloro-3-[substituted (amino/phenylamino)]-2-phenyl quinazolin-4 (3H)-one/thione derivatives and 1-(7-chloro-4-oxo/-2phenylquinazoline-3 (4H-yl)) substituted urea derivatives were prepared. The reaction scheme proceeds through the intermediate 7-chloro-2phenyl-4H-benzo[d] $[1,3]$ oxazin-4-one. The structures of the newly synthesised compounds were characterized from infrared (IR), ${ }^{1} \mathrm{H}$ nuclear magnetic resonance (NMR) and mass spectra (m/z) and elemental analysis. The anti-convulsant and CNS depressant activity were investigated by maximum electroshock (MES) seizure test and porsolt's behavioural despair test (forced swimming) respectively. The rota-rod test was performed to assess any probable changes in motor coordination induced by the test compounds.

Results: The physicochemical and spectroscopic data clearly confirmed the synthesis of quinazoline derivatives with a common skeleton. The synthesised compounds were evaluated for their anticonvulsant and CNS depressant properties. Among them, six compounds (IIc, IIg, IIj, IIIc, IIIg, IIIj) exhibited a good activity profile in CNS depressant activity. Five compounds (IIc, IIg, IIj, IIIg, IIIh) showed protection against MES-induced seizures.

Conclusion: The Quinazoline derivatives obtained from this research work indicates that the methyl/methoxy group in phenyl ring, amine and thiourea substitution at $3^{\text {rd }}$ position of quinazoline derivatives are essential for CNS depressant activity as well as anticonvulsant activity. Compounds IIc, IIg, IIj, IIIc, IIIg, IIIj, and IIIh were found to be a potent compound which may be effective as a potential source for the development of CNS depressant and anti-convulsant drugs with lesser side effects.

Keywords: 7-chloro-2-phenyl-4H-benzo[d][1,3]oxazin-4-one, Quinazoline derivatives, Anticonvulsant activity, CNS depressant activity

(C) 2017 The Authors. Published by Innovare Academic Sciences Pvt Ltd. This is an open access article under the CC BY license (http://creativecommons.org/licenses/by/4. 0/] DOI: http://dx.doi.org/10.22159/ijpps.2017v9i1.15492

\section{INTRODUCTION}

Epilepsy is a chronic neurological disorder characterised by unprovoked seizures and affects at least 1 percent (50 million) people worldwide $[1,2]$. Anti-convulsants are among the most widely utilised drugs for the treatment of CNS disorders [3]. Both dose related and idiosyncratic adverse effects are relatively common with the existing antiepileptic drugs [4] and this, together with their potential teratogenicity and drug interactions, make long-term use problematical. About one-third of patients do not respond well to current multiple drugs therapy [5]. Currently, no single drug is available which is effective against the various forms and degrees of convulsive disorders [6]. The anti-epileptic drugs mainly act through different mechanisms such as (a) enhancement of gamma amino butyric acid (GABA) mediated inhibition or another effect on the GABA system, (b) modulation of voltage-dependent $\mathrm{Na}^{+} / \mathrm{Ca}^{2+}$ channels, (c) modulation of synaptic release, (d) inhibition of synaptic excitation mediated by ionotropic glutamate receptors [7]. Several studies reveal that $63 \%$ of patients diagnosed and treated seizure-free and more than $50 \%$ of epilepsy patients have experienced unwanted side effects [8-10]. Hence, there is a need for safer, more effective anti-epileptic drugs for both generalised and partial seizures. So the search for new anti-epileptic drugs with lower toxicities and fewer side effects than the existing drugs is continuing. Quinazoline derivatives have been focused recently in the design of novel anti-convulsant and CNS depressant agents [11, 12] suggested that the presence of aryl hydrophobic binding site, hydrogen bonding domain and electron donor group regulate the pharmacokinetic properties of the anticonvulsant drug. Fig. 1 shows the pictorial representation of proposed designed quinazoline scaffold which bears the above-mentioned functional groups. The attachment of different functional groups to the quinazoline scaffold give the different structural activity relationship i.e. presence of aromatic/aliphatic group at position 2 and substituted aromatic ring at position 3 are essential for anti-convulsant and CNS depressant activity. As the aromatic ring increases the lipophilicity, the drug can cross the blood-brain barrier. The methyl/methoxy group substitution in the aromatic ring may increase the CNS activity. The introduction of electron withdrawing group at ortho/para position in aromatic ring may increase the CNS activity [13]. So, based on these mentioned hypotheses, the present research work focuses on the following objectives: (a) design and synthesis of some hybrid compounds having above mentioned molecular features, (b) anticonvulsant screening of the synthesised compounds by MES method, (c) CNS depressant screening by forced swimming test method.

In the present study, we also attempt to design and synthesise isomeric new series of quinazoline-4-one/4-thione derivatives, based on the pharmacophoric model of CNS activity by structural modifications retaining the essential structural features for the activity and evaluated for their anticonvulsant and CNS depressant properties.

\section{MATERIALS AND METHODS}

General

The synthesis of the target compounds was accomplished as illustrated in the fig. 2 . The compounds were synthesised according to the procedure given in the respective literature [14-16]. All the reagents and solvents used in the study were of analytical grade purity and procured from Sigma-Aldrich Pvt. Ltd. (India). The progress of the reaction was monitored by thin layered 
chromatography with hexane: ethyl acetate (3:2) as the mobile phase and performed on silica gel $60 \mathrm{~F}_{254}$ aluminium sheets (Merck Ltd., Germany); the products were purified by recrystallization. Melting points were determined in open capillaries using Stuart SMP10 (Barloworld scientific Ltd., UK), electro thermal melting point apparatus. IR spectra were recorded on Shimadzu 8400S FTIR (Shimadzu Corporation, Japan) spectrophotometer using was recorded in $\mathrm{cm}^{-1}$. $\mathrm{H}^{1} \mathrm{NMR}(400.13 \mathrm{MHz})$ spectra were acquired on a Bruker Advance II-400 NMR spectrophotometer using tetramethylsilane (TMS) as the internal standard, and the chemical shifts were recorded in $\delta$. The mass spectrum was obtained on Water ZQ4000 mass spectrophotometer. Elemental analysis for $\mathrm{C}, \mathrm{H}$ and $\mathrm{N}$ have performed on Perkin Elmer 2400 Series-II CHN analyser.

\section{Chemical synthesis}

\section{General procedure}

Synthesis of 7-chloro-2-phenyl-4H-benzo[d] [1, 3] oxazin-4-one (I): (Intermediate)

4-chloroanthranilic acid $(0.01 \mathrm{~mol})$ was dissolved in dry pyridine $(30 \mathrm{ml})$ by stirring slowly at room temperature. The solution was cooled to $0{ }^{\circ} \mathrm{C}$ and a solution of benzoyl chloride $(0.02 \mathrm{~mol})$ in dry pyridine $(30 \mathrm{ml})$ was added slowly with constant stirring. After this addition, the reaction mixture was further stirred for half an hour at room temperature and set aside for $1 \mathrm{hr}$. The pasty mass obtained was diluted with water (50 ml) and treated with aqueous sodium bicarbonate solution. When the effervescence ceased, the precipitate obtained was filtered off and washed with water, dried and recrystallized from diluted ethanol [14].

\section{General procedure for the synthesis of compounds, IIa-IIj}

7-chloro-2-phenyl-4H-benzo[d][1,3]oxazin-4-one $(0.01 \mathrm{~mol})$ and substituted phenylhydrazine derivatives/hydrazine hydrate/ semicarbazide/thiosemicarbazide $(0.01 \mathrm{~mol})$ were refluxed for $3 \mathrm{~h}$ in presence of glacial acetic acid. The reaction mixture was allowed to cool at room temperature. The crude product was recrystallized using absolute alcohol to afford the target compounds IIa-IIj [15].

\section{General procedure for the synthesis of compounds, IIIa-IIIj}

A mixture of 7-chloro-(3-amino/substituted phenylamino)-2-phenyl quinazoline-4 (3H)-one/1-(7-chloro-4-oxo-2-phenylquinazolin-3 (4H)-yl-urea/thiourea (10 mmol, $2.70 \mathrm{~kg})$ and phosphorus pentasulphide (1 mmol, $2.43 \mathrm{~g}$ ) was heated under reflux in anhydrous xylene $(100 \mathrm{ml})$ for $12 \mathrm{~h}$. The reaction mixture was filtered while hot, the solvent was evaporated and the residue was triturated with dimethyl sulphoxide $(10 \mathrm{ml})$ and filtered. The clear filtrate was poured into ice water, dried and recrystallised from ethanol to afford the target compounds IIIa-IIIj [16].

\section{Compound I (7-chloro-2-phenyl-4H-benzo[d] [1, 3] oxazin-4-one)}

White powder (methanol); 4-chloroanthranilic acid (0.01 mol) was dissolved in dry pyridine $(30 \mathrm{ml})$ by stirring slowly at room temperature. The solution was cooled to $0{ }^{\circ} \mathrm{C}$ and a solution of benzoyl chloride $(0.02 \mathrm{~mol})$ in dry pyridine $(30 \mathrm{ml})$ was added slowly with constant stirring. After this addition, the reaction mixture was further stirred for half an hour at room temperature and set aside for $1 \mathrm{~h}$. The pasty mass obtained was diluted with water $(50 \mathrm{ml})$ and treated with aqueous sodium bicarbonate solution. When the effervescence ceased, the precipitate obtained was filtered off and washed with water, dried and recrystallized from diluted ethanol as white solid. (Yield: $61.2 \%)$; m. p 156-158 ${ }^{\circ} \mathrm{C}$; IR $\left(\mathrm{cm}^{-1}\right) v_{\max }$ : Ar-CHstretch $\left(3072 \mathrm{~cm}^{-1}\right), \mathrm{C}=\mathrm{O}\left(1751.33 \mathrm{~cm}^{-1}\right), \mathrm{C}=\mathrm{N}\left(1592 \mathrm{~cm}^{-1}\right)$, cyclic C-O-Cstretch (1060.47 $\left.\mathrm{cm}^{-1}\right), \mathrm{C}-\mathrm{Cl}\left(680.72 \mathrm{~cm}^{-1}\right)$; ${ }^{1} \mathrm{H}$ NMR (DMSO-d6, 400.13MHz): $\delta 7.31-7.69$ (m, 5H, Ar-H,), 7.52-8.2 (t, 5H, Ar-H); MS m/z: $262.12\left(\mathrm{M}+\right.$ ), $\mathrm{C}_{14} \mathrm{H}_{8} \mathrm{ClNO}_{2}$ (Calcd. 257.67); Anal calcd. (\%) C, 65.26; $\mathrm{H}$, 3.13; N, 5.44; Found: C, 65.67; H, 3.52; N, 5.84.

Compound II $_{\mathrm{a}}$ (7-chloro-2-phenyl-3-(phenylamino) quinazolin4 (3H)-one)

Reddish brown crystalline solid (methanol); 7-chloro-2-phenyl-4Hbenzo[d][1,3]oxazin-4-one $(0.01 \mathrm{~mol})$ and phenyl hydrazine derivatives hydrate $(0.01 \mathrm{~mol})$ were refluxed for $3 \mathrm{~h}$ in presence of glacial acetic acid. The reaction mixture was allowed to cool at room temperature. The crude product was recrystallized using absolute alcohol as reddish brown solid. (Yield: $87.2 \%$ ); m. p. $135-145^{\circ} \mathrm{C}$; IR $\left(\mathrm{cm}^{-1}\right) v_{\max } \mathrm{Ar}-\mathrm{CH}_{\text {stretch }}\left(3262 \mathrm{~cm}^{-1}\right), \mathrm{C}=0\left(1664.49 \mathrm{~cm}^{-1}\right), \mathrm{C}=\mathrm{N}(1595$ $\left.\mathrm{cm}^{-1}\right)$, N-NH ${ }_{\text {stretch }}\left(3343.75 \mathrm{~cm}^{-1}\right), \mathrm{C}-\mathrm{Cl}\left(689.04 \mathrm{~cm}^{-1}\right),{ }^{1} \mathrm{H}$ NMR (DMSO$\left.\mathrm{d}_{6}, 400.13 \mathrm{MHz}\right), \delta$ 7.491-7.601 (m, 5H, Ar-H,) 7.781-8.115 (m, 5H, Ar-H), 7.907-8.723 (t, 3H, Ar-H), 3.466 (s, 1H, N-H); MS m/z: 365.11 (M+); $\mathrm{C}_{20} \mathrm{H}_{14} \mathrm{ClN}_{3} \mathrm{O}$ (Calcd. 347.8); Anal calcd. (\%) C, 69.07; H, 4.06; N, 12.08; Found: C, 69.48;H, 4.48;N, 12.48 .

\section{Compound $\mathrm{II}_{\mathrm{b}}$ (7-chloro-2-phenyl-3-(o-chloro-phenylamino)} quinazolin-4 (3H)-one)

White crystalline solid (methanol); 7-chloro-2-phenyl-4H-benzo [d] [1, 3] oxazin-4-one $(0.01 \mathrm{~mol})$ and 2-chloro phenyl hydrazine $(0.01 \mathrm{~mol})$ were refluxed for $3 \mathrm{~h}$ in presence of glacial acetic acid. The reaction mixture was allowed to cool at room temperature. The crude product was recrystallized using absolute alcohol as white crystalline solid. (Yield: 57. 14\%); m. p 168-170 ${ }^{\circ} \mathrm{C}$; IR $\left(\mathrm{cm}^{-1}\right) v_{\max } \mathrm{Ar}-$ $\mathrm{CH}_{\text {stretch }}\left(3323.43 \mathrm{~cm}^{-1}\right), \mathrm{C}=\mathrm{O}\left(1665.77 \mathrm{~cm}^{-1}\right), \mathrm{C}=\mathrm{N}\left(1592 \mathrm{~cm}^{-1}\right), \mathrm{N}-$ $\mathrm{NH}_{\text {stretch }}\left(3307.746 \mathrm{~cm}^{-1}\right), \mathrm{C}-\mathrm{Cl}\left(676.27 \mathrm{~cm}^{-1}\right),{ }^{1} \mathrm{H}$ NMR (DMSO-d 6 , 400 . $13 \mathrm{MHz}), \delta$ 6.753-7.181 (m, 4H, Ar-H,), 7.305-7.72 (t, 5H, Ar-H), 7.527.907 (t, 3H, Ar-H), 4.56 (s, 1H, N-H), MS, m/z: $382.02(\mathrm{M}+$ ); $\mathrm{C}_{20} \mathrm{H}_{13} \mathrm{C}_{12} \mathrm{~N}_{3} \mathrm{O}$ (Calcd. 382.24); Anal calcd. (\%)C, 62.84; $\mathrm{H}, 3.43 ; \mathrm{N}$, 10.99; Found: C, 63.24;H, 3.85;N, 11.35 .

Compound II (7-chloro-2-phenyl-3-(o-methyl-phenylamino) quinazolin-4 (3H)-one)

Light brown crystalline solid (methanol); 7-chloro-2-phenyl-4Hbenzo[d][1,3]oxazin-4-one $(0.01 \mathrm{~mol})$ and 2-methyl phenyl hydrazine $(0.01 \mathrm{~mol})$ were refluxed for $3 \mathrm{~h}$ in presence of glacial acetic acid. The reaction mixture was allowed to cool at room temperature. The crude product was recrystallized using absolute alcohol as Light brown crystalline solid. (Yield: 67\%); m. p 163-166 ${ }^{\circ} \mathrm{C}$; IR $\left(\mathrm{cm}^{-1}\right) v_{\max } \mathrm{Ar}-$ $\mathrm{CH}_{\text {stretch }}\left(3242.17 \mathrm{~cm}^{-1}\right), \mathrm{C}=\mathrm{O}\left(1677.66 \mathrm{~cm}^{-1}\right), \mathrm{C}=\mathrm{N}\left(1654 \mathrm{~cm}^{-1}\right), \mathrm{N}-$ $\mathrm{NH}_{\text {stretch }}\left(3309.67 \mathrm{~cm}^{-1}\right), \mathrm{C}^{-\mathrm{CH}_{3}}\left(2911.79 \mathrm{~cm}^{-1}\right),{ }^{1} \mathrm{H}$ NMR (DMSO-d6, 400.13MHz), $\delta$ 6.85-7.02 (m, 4H, Ar-H), 7.282-7.621 (m, 5H, Ar-H), 7.653-7.91 (t, 3H, Ar-H), 3.818 (s, 1H, N-H), 2.51 (s, 1H, Ar-CH3), MS, m/z: $361.23(\mathrm{M}+) ; \mathrm{C}_{20} \mathrm{H}_{13} \mathrm{Cl}_{2} \mathrm{~N}_{3} \mathrm{O}$ (Calcd. 361.82); Anal calcd. (\%) C, 69.71; H, 4.46; N, 11.61; Found: C, 70.12; H, 4.87; N, 12.04.

Compound $\quad I_{d}$ (7-chloro-2-phenyl-3-(p-chloro-phenylamino) quinazolin-4 (3H)-one)

Brown crystalline solid (methanol); 7-chloro-2-phenyl-4H-benzo[d] $[1,3]$ oxazin-4-one $(0.01 \mathrm{~mol})$ and $p$-chloro phenyl hydrazine $(0.01$ mol) were refluxed for $3 \mathrm{~h}$ in presence of glacial acetic acid. The reaction mixture was allowed to cool at room temperature. The crude product was recrystallized using absolute alcohol as brown crystalline solid. (Yield: 91.67\%); m. p 171-174 ${ }^{\circ} \mathrm{C}$; IR $\left(\mathrm{cm}^{-1}\right) ; v_{\max } \mathrm{Ar}-$ $\mathrm{CH}_{\text {stretch }}\left(3010.02 \mathrm{~cm}^{-1}\right), \mathrm{C}=\mathrm{O}\left(1665.38 \mathrm{~cm}^{-1}\right), \mathrm{C}=\mathrm{N}\left(1594.74 \mathrm{~cm}^{-1}\right), \mathrm{N}-$ $\mathrm{NH}_{\text {stretch }}\left(3240.41 \mathrm{~cm}^{-1}\right), \mathrm{C}-\mathrm{Cl}\left(698 \mathrm{~cm}^{-1}\right), 1 \mathrm{H}$ NMR (DMSO-d6, 400.13MHz), $\delta$ 6. 743-7.23 (m, 4H, Ar-H,), 7. 352-7.68 (t, 4H, Ar-H), 7.54-7.907 (t, 3H, Ar-H), $4.43(\mathrm{~s}, 1 \mathrm{H}, \mathrm{N}-\mathrm{H}), \mathrm{MS}, \mathrm{m} / \mathrm{z}: 382.07(\mathrm{M}+)$; $\mathrm{C}_{20} \mathrm{H}_{13} \mathrm{Cl}_{2} \mathrm{~N}_{3} \mathrm{O}$ (Calcd. 382.24); Anal calcd. (\%) C, 62.84; H, 3.42; N, 10.99; Found: C, 63.14; H, 3.82; N, 11.12 .

Compound II $_{e}$ (7-chloro-2-phenyl-3-(p-bromo-phenylamino) quinazolin-4 (3H)-one)

Brown crystalline solid (methanol); 7-chloro-2-phenyl-4H-benzo[d] $[1,3]$ oxazin-4-one $(0.01 \mathrm{~mol})$ and p-bromo phenyl hydrazine $(0.01$ mol) were refluxed for $3 \mathrm{~h}$ in presence of glacial acetic acid. The reaction mixture was allowed to cool at room temperature. The crude product was recrystallized using absolute alcohol as brown crystalline solid. (Yield: 91.67\%); m. p 172-175 ${ }^{\circ} \mathrm{C} ; \lambda \max (\mathrm{nm}) 274$; IR ( $\left.\mathrm{cm}^{-1}\right)$; vmax Ar-CH stretch $\left(3271.23 \mathrm{~cm}^{-1}\right), \mathrm{C}=\mathrm{O}\left(1691.23 \mathrm{~cm}^{-1}\right), \mathrm{C}=\mathrm{N}$ $\left(1645.30 \mathrm{~cm}^{-1}\right), \mathrm{N}-\mathrm{NH}_{\text {stretch }}\left(3332.01 \mathrm{~cm}^{-1}\right), \mathrm{C}-\mathrm{Br}\left(693.97 \mathrm{~cm}^{-1}\right),{ }^{1} \mathrm{H}$ NMR (DMSO-d6, 400.13MHz), $\delta$ 6. 52-7.24 (m, 5H, Ar-H), 7. 43-7.62 (m, 4H, Ar-H), 7.42-7.85 (m, 3H, Ar-H), 4.42 (s, 1H, N-H), MS, m/z: 412.85 (M+); $\mathrm{C}_{20} \mathrm{H}_{13} \mathrm{Br} \mathrm{ClN}_{3} \mathrm{O}$ (Calcd. 426.69); Anal calcd. (\%) C, 56.3; H, 3.07; N, 9.85; Found: C, 56.72; H, 3.48; N, 10.25.

Compound IIf (7-chloro-2-phenyl-3-(p-nitro-phenylamino) quinazolin-4 (3H)-one)

Reddish brown solid (methanol); 7-chloro-2-phenyl-4H-benzo[d][1, 3] oxazin-4-one $(0.01 \mathrm{~mol})$ and $\mathrm{p}$-nitro phenyl hydrazine $(0.01 \mathrm{~mol})$ 
were refluxed for $3 \mathrm{~h}$ in presence of glacial acetic acid. The reaction mixture was allowed to cool at room temperature. The crude product was recrystallized using absolute alcohol as brown crystalline solid. Yield: $80 \%)$; m. p 171-173 ${ }^{\circ} \mathrm{C}$; IR $\left(\mathrm{cm}^{-1}\right)$; $v_{\max } \mathrm{Ar}-$ $\mathrm{CH}_{\text {stretch }}\left(3250 \mathrm{~cm}^{-1}\right), \mathrm{C}=\mathrm{O}\left(1650.49 \mathrm{~cm}^{-1}\right), \mathrm{C}=\mathrm{N}\left(1592 \mathrm{~cm}^{-1}\right), \mathrm{N}-\mathrm{NH}_{\text {stretch }}$ (3341.75 cm-1), C-Cl $\left(682.04 \mathrm{~cm}^{-1}\right),{ }^{1} \mathrm{H}$ NMR (DMSO-d $6,400.13 \mathrm{MHz}$ ), $\delta$ 6. 94-7.31 (t, 5H, Ar-H), 6.96-8.12 (m, 5H, Ar-H), 7.5-7.81 (t, 3H, ArH), $3.9(\mathrm{~s}, 1 \mathrm{H}, \mathrm{N}-\mathrm{H}), \mathrm{MS}, \mathrm{m} / \mathrm{z}: 378.15(\mathrm{M}+) ; \mathrm{C}_{20} \mathrm{H}_{13} \mathrm{ClN}_{4} \mathrm{O}_{3}$ (Calcd. 392.8); Anal calcd. (\%) C, 61.16; H, 3.34; N, 14.26; Found: C, 62.12; H, $4.15 ; \mathrm{N}, 14.67$.

\section{Compound IIg (7-chloro-2-phenyl-3-(p-methoxy-phenylamino) quinazolin-4 (3H)-one)}

Brownish yellow solid (methanol); 7-chloro-2-phenyl-4H-benzo[d] $[1,3]$ oxazin-4-one $(0.01 \mathrm{~mol})$ and p-methoxy phenyl hydrazine $(0$. $01 \mathrm{~mol}$ ) were refluxed for $3 \mathrm{~h}$ in presence of glacial acetic acid. The reaction mixture was allowed to cool at room temperature. The crude product was recrystallized using absolute alcohol as brown yellow solid. Yield: 92\%); m. p 170-173 ${ }^{\circ} \mathrm{C}$; IR $\left(\mathrm{cm}^{-1}\right) v_{\max } \mathrm{Ar}-\mathrm{CH}_{\text {stretch }}$ $\left(3114.98 \mathrm{~cm}^{-1}\right), \mathrm{C}=\mathrm{O}\left(1752.81 \mathrm{~cm}^{-1}\right), \mathrm{C}=\mathrm{N}\left(1664.02 \mathrm{~cm}^{-1}\right), \mathrm{N}-\mathrm{NH}_{\text {stretch }}$ (3310.40 $\left.\mathrm{cm}^{-1}\right), \mathrm{OCH}_{3}-\mathrm{CH}_{\text {stretch }}\left(3008.64 \mathrm{~cm}^{-1}\right), \mathrm{Ar}-\mathrm{CH}_{\text {stretch }}$ (3271.23 $\mathrm{cm}^{-1}$ ), 1H NMR (DMSO-d6, 400.13MHz), $\delta$ 6.5-6.7 (m, 4H, Ar-H), 7.28$7.62(\mathrm{~m}, 5 \mathrm{H}, \mathrm{Ar}-\mathrm{H}), 7.46-7.921(\mathrm{t}, 3 \mathrm{H}, \mathrm{Ar}-\mathrm{H}), 4.1(\mathrm{~s}, 1 \mathrm{H}, \mathrm{N}-\mathrm{H}), 3.78(\mathrm{~s}$ $1 \mathrm{H}, \mathrm{Ar}-\mathrm{OCH}_{3}$ ), MS, m/z: $345.12(\mathrm{M}+) ; \mathrm{C}_{21} \mathrm{H}_{16} \mathrm{ClN}_{3} \mathrm{O}$ (Calcd. 361.82); Anal Calcd. (\%) C, 66.76; H, 4.27; N, 11.12; Found: C, 67.16; H, 4.67; $\mathrm{N}, 11.54$.

\section{Compound $\mathrm{II}_{\mathrm{h}}$ (3-amino-7-chloro-2-phenyquinazolin-4-(3H)- one)}

Brown crystalline solid (methanol); 7-chloro-2-phenyl-4H-benzo[d] $[1,3]$ oxazin-4-one $(0.01 \mathrm{~mol})$ and hydrazine hydrazine hydrate $(0.01 \mathrm{~mol})$ were refluxed for $3 \mathrm{~h}$ in presence of glacial acetic acid. The reaction mixture was allowed to cool at room temperature. The crude product was recrystallized using absolute alcohol as brown yellow solid. (Yield: 92\%); m. p 170-173 ${ }^{\circ} \mathrm{C}$; $\lambda \max (\mathrm{nm}) 278$; IR (cm 1); vmax Ar- $\mathrm{CH}_{\text {stretch }}\left(3126.37 \mathrm{~cm}^{-1}\right), \mathrm{C}=0$ (1598 $\left.\mathrm{cm}^{-1}\right), \mathrm{C}=\mathrm{N}(1552.02$ $\left.\mathrm{cm}^{-1}\right), \mathrm{N}-\mathrm{NH}_{\text {stretch }}\left(3283.49 \mathrm{~cm}^{-1}\right), \mathrm{C}-\mathrm{Cl}\left(696 \mathrm{~cm}^{-1}\right), 1 \mathrm{H}$ NMR (DMSO-d6, 400.13MHz), $\delta$ 7.21-7.56 (m, 5H, Ar-H), 7.42-7.87 (m, 3H, Ar-H), 2.4 (s, 1H, N-H), MS, m/z: $272.12\left(\mathrm{M}+\right.$ ); $\mathrm{C}_{14} \mathrm{H}_{10} \mathrm{ClN}_{3} \mathrm{O}$ (Calcd. 271.7); Anal calcd. (\%) C, 61.89; H, 3.71; N, 15.47; Found: C, 62.29; H, 4.12; N 15.87 .

\section{Compound $\mathrm{II}_{\mathrm{i}}$ (1-(7-chloro-4-oxo-2-phenylquinazoline-3 (4H- yl)) urea)}

White crystalline solid (methanol); 7-chloro-2-phenyl-4H-benzo[d] $[1,3]$ oxazin-4-one $(0.01 \mathrm{~mol})$ and semicarbazide $(0.01 \mathrm{~mol})$ were refluxed for $3 \mathrm{~h}$ in presence of glacial acetic acid. The reaction mixture was allowed to cool at room temperature. The crude product was recrystallized using absolute alcohol as white crystalline solid. (Yield: $60 \%$ ); m. p $165-167{ }^{\circ} \mathrm{C}$; IR $\left(\mathrm{cm}^{-1}\right) v_{\max } \mathrm{Ar}-$ $\mathrm{CH}_{\text {stretch }}\left(3199.89 \mathrm{~cm}^{-1}\right), \mathrm{C}=\mathrm{O}\left(1671.85 \mathrm{~cm}^{-1}\right), \mathrm{C}=\mathrm{N}\left(1593.8 \mathrm{~cm}^{-1}\right), \mathrm{N}-$ $\mathrm{NH}_{\text {stretch }}\left(3027.51 \mathrm{~cm}^{-1}\right), \mathrm{C}-\mathrm{Cl}\left(692.51 \mathrm{~cm}^{-1}\right),{ }^{1} \mathrm{H}$ NMR (DMSO-d6, 400.13MHz), $\delta$ 7.25-7.58 (m, 5H, Ar-H), 7.37-7.86 (m, 3H, Ar-H), 5.8 (s, $1 \mathrm{H}, \mathrm{N}-\mathrm{H}$ ), MS, m/z: $312.45\left(\mathrm{M}^{+}\right) ; \mathrm{C}_{15} \mathrm{H}_{11} \mathrm{ClN}_{4} \mathrm{O}_{2}$ (Calcd. 314.72); Anal calcd. (\%) C, 57.24; H, 3.52; N, 17.8; Found: C, 57.46; H, 3.94; N, 18.21.

\section{Compound $\mathrm{II}_{\mathrm{j}}$ (1-(7-chloro-4-oxo-2-phenylquinazoline-3 (4H- yl))thiourea)}

White amorphous solid (methanol); 7-chloro-2-phenyl-4H-benzo[d] $[1,3]$ oxazin-4-one $(0.01 \mathrm{~mol})$ and thiosemicarbazide $(0.01 \mathrm{~mol})$ were refluxed for $3 \mathrm{~h}$ in presence of glacial acetic acid. The reaction mixture was allowed to cool at room temperature. The crude product was recrystallized using absolute alcohol as white crystalline solid. (Yield: $70 \%$ ); m. p $162-165^{\circ} \mathrm{C}$; IR (cm-1); $v_{\max } \mathrm{Ar}$ $\mathrm{CH}_{\text {stretch }}\left(3126.71 \mathrm{~cm}^{-1}\right), \mathrm{C}=\mathrm{O}\left(1644.7 \mathrm{~cm}^{-1}\right), \mathrm{C}=\mathrm{N}\left(1552.32 \mathrm{~cm}^{-1}\right), \mathrm{N}-$ $\mathrm{NH}_{\text {stretch }}\left(3432.52 \mathrm{~cm}^{-1}\right), \mathrm{C}-\mathrm{Cl}\left(755.72 \mathrm{~cm}^{-1}\right){ }^{1} \mathrm{H}$ NMR (DMSO-d6, 400.13MHz), $\delta$ 7.24-7.5 (m, 5H, Ar-H), 7.32-7.78 (m, 3H, Ar-H), 2.3 (s, 1H, N-H), MS, m/z: 332. $15\left(\mathrm{M}+\right.$ ); $\mathrm{C}_{15} \mathrm{H}_{11} \mathrm{ClN}_{4} \mathrm{OS}$ (Calcd. 330.79); Anal calcd. (\%) C, 54.46; H, 3.35; N, 16.94; Found: C, 54.86; H, 3.35; $\mathrm{N}, 16.94$.

\section{Compound IIIa (7-chloro-2-phenyl-3-(phenylamino)-quinazolin- 4 (3H)-thione)}

Light brown crystalline solid (methanol) A mixture of 7-chloro-2phenyl-3-(phenylamino) quinazolin-4 $(3 \mathrm{H})$-one (10 mmol, $2.70 \mathrm{~g})$ and phosphorus penta sulphide ( $1 \mathrm{mmol}, 2.43 \mathrm{~g}$ ) was heated under reflux in anhydrous xylene $(100 \mathrm{ml})$ for $12 \mathrm{~h}$. The reaction mixture was filtered while hot, the solvent was evaporated and the residue was triturated with dimethyl sulphoxide $(10 \mathrm{ml})$ and filtered. The clear filtrate was poured into ice water, dried and recrystallised from ethanol to form light brown crystalline solid. (Yield: 68\%); $\mathrm{m}$. p. $165-168{ }^{\circ} \mathrm{C}$; IR (cm-1) $v_{\max } \mathrm{Ar}-\mathrm{CH}_{\text {stretch }}\left(3025 \mathrm{~cm}^{-1}\right), \mathrm{C}-\mathrm{N}(1150.39$ $\left.\mathrm{cm}^{-1}\right), \mathrm{C}=\mathrm{N}\left(1666.18 \mathrm{~cm}^{-1}\right), \mathrm{C}=\mathrm{S}\left(1262.41 \mathrm{~cm}^{-1}\right), \mathrm{C}-\mathrm{Cl}\left(735.15 \mathrm{~cm}^{-1}\right), \mathrm{N}-$ $\mathrm{NH}$ (bend) (3057.18 $\left.\mathrm{cm}^{-1}\right),{ }^{1} \mathrm{H}$ NMR (DMSO-d6, 400.13MHz), $\delta 6.5-$ 7.4 (m, 5H, Ar-H), 6.42-7.71 (m, 5H, Ar-H), 7.15-7.5 (m, 3H, Ar-H), 3.8 (s, $1 \mathrm{H}, \mathrm{N}-\mathrm{H}$ ), MS, m/z: $362.15\left(\mathrm{M}^{+}\right) ; \mathrm{C}_{20} \mathrm{H}_{14} \mathrm{ClN}_{3} \mathrm{~S}$ (Calcd. 363.86); Anal calcd. (\%) C, 66.02; H, 3.88; N, 11.55; Found: C, 66.42; H, 4.28; N, 11.96.

Compound III (7-chloro-2-phenyl-3-(o-chlorophenylamino)quinazolin-4 (3H)-thione)

Brown crystalline solid (methanol); A mixture of 7-chloro-2-phenyl3-(o-chloro-phenylamino) quinazolin-4 (3H)-one (10 mmol, $2.70 \mathrm{~g}$ ) and phosphorus penta sulphide $(1 \mathrm{mmol}, 2.43 \mathrm{~g}$ ) was heated under reflux in anhydrous xylene $(100 \mathrm{ml})$ for $12 \mathrm{~h}$. The reaction mixture was filtered while hot, the solvent was evaporated and the residue was triturated with dimethyl sulphoxide $(10 \mathrm{ml})$ and filtered. The clear filtrate was poured into ice water, dried and recrystallised from ethanol to form brown crystalline solid. (Yield: 67\%); m. p. 140-142 ${ }^{\circ} \mathrm{C}$; IR $\left(\mathrm{cm}^{-1}\right)$; $v_{\max } \mathrm{Ar}-\mathrm{CH}_{\text {stretch }}\left(3010.02 \mathrm{~cm}^{-1}\right), \mathrm{C}=\mathrm{S}(1665.38$ $\left.\mathrm{cm}^{-1}\right), \mathrm{C}=\mathrm{N}\left(1594.74 \mathrm{~cm}^{-1}\right), \mathrm{N}-\mathrm{NH}_{\text {stretch }}\left(3240.41 \mathrm{~cm}^{-1}\right), \mathrm{C}-\mathrm{Cl}\left(698 \mathrm{~cm}^{-1}\right)$, ${ }^{1} \mathrm{H}$ NMR (DMSO-d6, 400.13MHz), $\delta$ 6.62-7.35 (m, 5H, Ar-H), 6.457.68 (m, 5H, Ar-H), 7.2-7.48 (m, 3H, Ar-H), 4.1 (s, 1H, N-H), MS, m/z: $396.14\left(\mathrm{M}+\right.$ ); $\mathrm{C}_{14} \mathrm{H}_{10} \mathrm{ClN}_{3} \mathrm{~S}$ (Calcd. 398.31); Anal calcd. (\%) C, 60.31; H, 3.29; N, 10.55; Found: C, 60.71; H, 3.59; N, 10.97 .

Compound III ${ }_{c}$ (7-chloro-2-phenyl-3-(o-methylphenylamino)quinazolin-4 (3H)-thione)

Brownish yellow crystalline solid (methanol); A mixture of 7-chloro2-phenyl-3-(o-methyl-phenylamino) quinazolin-4 (3H)-one $(10$ mmol, $2.70 \mathrm{~g})$ and phosphorus penta sulphide $(1 \mathrm{mmol}, 2.43 \mathrm{~g})$ was heated under reflux in anhydrous xylene $(100 \mathrm{ml})$ for $12 \mathrm{~h}$. The reaction mixture was filtered while hot,the solvent was evapourated and the residue was triturated with dimethyl sulphoxide $(10 \mathrm{ml})$ and filtered. The clear filtrate was poured into ice water, dried and recrystallised from ethanol to form brownish yellow crystalline solid. (Yield: $71 \%$ ); m. p 172-175 ${ }^{\circ} \mathrm{C}$; IR $\left(\mathrm{cm}^{-1}\right) ; v_{\max } \mathrm{Ar}^{-\mathrm{CH}_{\text {stretch }}}$ (3114.98 cm-1), C=S $\left(1264.56 \mathrm{~cm}^{-1}\right), \mathrm{C}=\mathrm{N}\left(1664.02 \mathrm{~cm}^{-1}\right), \mathrm{C}-\mathrm{N}$ $\left(1099.72 \mathrm{~cm}^{-1}\right) \mathrm{N}-\mathrm{NH}_{\text {stretch }}\left(3310.40 \mathrm{~cm}^{-1}\right), \mathrm{CH}_{3}-\mathrm{CH}_{\text {stretch }}(3008.64 \mathrm{~cm}$ 1), ${ }^{1} \mathrm{H}$ NMR (DMSO-d6, 400.13MHz), $\delta 6.5-7.57(\mathrm{~m}, 5 \mathrm{H}, \mathrm{Ar}-\mathrm{H}), 6.67-$ $7.58(\mathrm{~m}, 5 \mathrm{H}, \mathrm{Ar}-\mathrm{H}), 7.31-7.35(\mathrm{t}, 3 \mathrm{H}, \mathrm{Ar}-\mathrm{H}), 3.9(\mathrm{~s}, 1 \mathrm{H}, \mathrm{N}-\mathrm{H}), 2.34(\mathrm{~s}$, $1 \mathrm{H}, \mathrm{Ar}-\mathrm{CH} 3), \mathrm{MS}, \mathrm{m} / \mathrm{z}: 378.19\left(\mathrm{M}+\mathrm{C}_{20} \mathrm{H}_{13} \mathrm{Cl}_{2} \mathrm{~N}_{3} \mathrm{~S}\right.$ (Calcd. 377.89); Anal calcd. (\%) C, 66.75; H, 4.27; N, 10.55; Found: C, 60.71; H, 3.47; $\mathrm{N}, 11.44$.

Compound III (7-chloro-2-phenyl-3-(p-chlorophenylamino)quinazolin-4 (3H)-thione)

Dark brown crystalline solid (methanol); A mixture of 7-chloro-2phenyl-3-(p-chloro-phenylamino) quinazolin-4 (3H)-one $(10 \mathrm{mmol}$, $2.70 \mathrm{~g})$ and phosphorus penta sulphide $(1 \mathrm{mmol}, 2.43 \mathrm{~g})$ was heated under reflux in anhydrous xylene $(100 \mathrm{ml})$ for $12 \mathrm{~h}$. The reaction mixture was filtered while hot, the solvent was evaporated and the residue was triturated with dimethyl sulphoxide $(10 \mathrm{ml})$ and filtered. The clear filtrate was poured into ice water, dried and recrystallised from ethanol to form dark brown crystalline solid. (Yield: 72\%); m. p 162-163 ${ }^{\circ} \mathrm{C}$; IR $\left(\mathrm{cm}^{-1}\right) v_{\max } \mathrm{Ar}-\mathrm{CH}_{\text {stretch }}\left(3029 \mathrm{~cm}^{-1}\right)$, C-N (1171.25 $\left.\mathrm{cm}^{-1}\right), \mathrm{C}=\mathrm{N}\left(1676.63 \mathrm{~cm}^{-1}\right), \mathrm{C}=\mathrm{S}\left(1263.99 \mathrm{~cm}^{-1}\right), \mathrm{C}-\mathrm{Cl}$ (761.37 $\left.\mathrm{cm}^{-1}\right), \mathrm{N}-\mathrm{NH}$ (bend) $\left(3196.89 \mathrm{~cm}^{-1}\right),{ }^{1} \mathrm{H}$ NMR (DMSO-d6, $400.13 \mathrm{MHz}), \delta 6.58-7.24(\mathrm{~m}, 5 \mathrm{H}, \mathrm{Ar}-\mathrm{H}), 6.7-7.34(\mathrm{~m}, 5 \mathrm{H}, \mathrm{Ar}-\mathrm{H}), 7.25-$ $7.42(\mathrm{~m}, 3 \mathrm{H}, \mathrm{Ar}-\mathrm{H}), 4.2(\mathrm{~s}, 1 \mathrm{H}, \mathrm{N}-\mathrm{H}), \mathrm{MS}, \mathrm{m} / \mathrm{z}: 398.05(\mathrm{M}+)$; $\mathrm{C}_{20} \mathrm{H}_{13} \mathrm{Cl}_{2} \mathrm{~N}_{3} \mathrm{~S}$ (Calcd. 398.31); Anal calcd. (\%) C, 60.31; H, 3.29; N, 11.12; Found: C, 67.12; H, 4.67; N, 11.02 . 
Compound IIIe (3-(4-bromophenylamino)-7-chloro-2-phenylquinazolin-4 (3H)-thione)

Brownish yellow crystalline solid (methanol); A mixture of 7-chloro-2phenyl-3-(p-bromo-phenylamino) quinazolin-4 (3H)-one $(10 \mathrm{mmol}$, $2.70 \mathrm{~g})$ and phosphorus penta sulphide $(1 \mathrm{mmol}, 2.43 \mathrm{~g})$ was heated under reflux in anhydrous xylene $(100 \mathrm{ml})$ for $12 \mathrm{~h}$. The reaction mixture was filtered while hot, the solvent was evaporated and the residue was triturated with dimethyl sulphoxide $(10 \mathrm{ml})$ and filtered. The clear filtrate was poured into ice water, dried and recrystallised from ethanol to form brownish yellow crystalline solid. (Yield: $69 \%$ ); m. p. $170-173^{\circ} \mathrm{C}$; IR $\left(\mathrm{cm}^{-1}\right) ; v_{\max }$ Ar-CH $\mathrm{CH}_{\text {stretch }}\left(2918.13 \mathrm{~cm}^{-1}\right), \mathrm{C}=\mathrm{S}\left(1233.04 \mathrm{~cm}^{-1}\right), \mathrm{C}=\mathrm{N}$

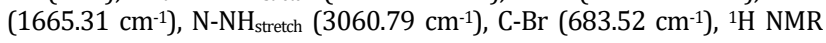
(DMSO-d6, 400.13MHz), $\delta 6.53-7.58(\mathrm{~m}, 5 \mathrm{H}, \mathrm{Ar}-\mathrm{H}$ ), 7.43-7.62 (m, 4H, ArH), 7.24-7.41 (m, 3H, Ar-H), $4.2(\mathrm{~s}, 1 \mathrm{H}, \mathrm{N}-\mathrm{H}), \mathrm{MS}, \mathrm{m} / \mathrm{z}: 445.82(\mathrm{M}+)$; $\mathrm{C}_{20} \mathrm{H}_{13} \mathrm{BrClN}_{3} \mathrm{~S}$ (Calcd. 442.76); Anal calcd. (\%) C, 54.25; H, 2.96; N, 9.49; Found: C, 54.65; H, 3.37; N, 9.59.

\section{Compound IIIf (7-chloro-2-phenyl-3-(p-nitrophenylamino)-} quinazolin-4 (3H)-thione)

Reddish brown crystalline solid (methanol); A mixture of 7-chloro2-phenyl-3-(p-nitro-phenylamino) quinazolin-4 (3H)-one $(10 \mathrm{mmol}$, $2.70 \mathrm{~g})$ and phosphorus penta sulphide $(1 \mathrm{mmol}, 2.43 \mathrm{~g})$ was heated under reflux in anhydrous xylene $(100 \mathrm{ml})$ for $12 \mathrm{~h}$. The reaction mixture was filtered while hot, the solvent was evaporated and the residue was triturated with dimethyl sulphoxide $(10 \mathrm{ml})$ and filtered. The clear filtrate was poured into ice water, dried and recrystallised from ethanol to form reddish brown crystalline solid. (Yield: 70\%); m. p 171-174 ${ }^{\circ} \mathrm{C}$; IR $\left(\mathrm{cm}^{-1}\right) v_{\max } A r-\mathrm{CH}_{\text {stretch }}\left(3255 \mathrm{~cm}^{-1}\right)$, $\mathrm{C}=0\left(1648.49 \mathrm{~cm}^{-1}\right), \mathrm{C}=\mathrm{N}\left(1594 \mathrm{~cm}^{-1}\right), \mathrm{N}-\mathrm{NH}_{\text {stretch }}\left(3342.75 \mathrm{~cm}^{-1}\right), \mathrm{C}-\mathrm{Cl}$ (692.04 $\mathrm{cm}^{-1}$ ), ${ }^{1} \mathrm{H}$ NMR (DMSO- $\left.\mathrm{d}_{6}, 400.13 \mathrm{MHz}\right), \delta 7.12-7.56(\mathrm{t}, 5 \mathrm{H}$, Ar-H,), 6.95-8.2 (m, 5H, Ar-H), 7.31-7.42 (t, 3H, Ar-H), 3.89 (s, 1H, N$\mathrm{H}), \mathrm{MS}, \mathrm{m} / \mathrm{z}: 407.16(\mathrm{M}+) ; \mathrm{C}_{20} \mathrm{H}_{13} \mathrm{ClN}_{4} \mathrm{O}_{2} \mathrm{~S}$ (Calcd. 408.86); Anal calcd. (\%) C, 58.75;H, 3.20; N, 13.7; Found: C, 59.12; H, 3.62; N, 14.12.

\section{Compound IIIg (7-chloro-2-phenyl-3-(p-methoxyphenylamino)-} quinazolin-4 (3H)-thione)

Light brown crystalline solid (methanol); A mixture of 7-chloro-2phenyl-3-(p-methoxy-phenylamino) quinazolin-4 (3H)-one (10 mmol, $2.70 \mathrm{~g})$ and phosphorus penta sulphide $(1 \mathrm{mmol}, 2.43 \mathrm{~g}$ ) was refluxed in anhydrous xylene $(100 \mathrm{ml})$ for $12 \mathrm{~h}$. The reaction mixture was filtered while hot, the solvent was evaporated and the residue was triturated with dimethyl sulphoxide $(10 \mathrm{ml})$ and filtered. The clear filtrate was poured into ice water, dried and recrystallised from ethanol to form light brown crystalline solid. (Yield: 72\%); m. p 172-174 ${ }^{\circ} \mathrm{C}$; IR $\left(\mathrm{cm}^{-1}\right) v_{\max } \mathrm{Ar}-\mathrm{CH}_{\text {stretch }}\left(3242.17 \mathrm{~cm}^{-}\right.$ $\left.{ }^{1}\right), C=S\left(1261.65 \mathrm{~cm}^{-1}\right), C=N\left(1677.66 \mathrm{~cm}^{-1}\right), C-\mathrm{N}\left(1193.01 \mathrm{~cm}^{-1}\right), \mathrm{N}-$ $\mathrm{NH}_{\text {stretch }}\left(3309.67 \mathrm{~cm}^{-1}\right), \mathrm{C}-\mathrm{CH}_{3}\left(2911.79 \mathrm{~cm}^{-1}\right),{ }^{1} \mathrm{H}$ NMR (DMSO-d6, $400.13 \mathrm{MHz}$ ), $\delta 6.54-7.52$ (m, 5H, Ar-H), 6.7-7.58 (m, 5H, Ar-H), 7.24$7.35(\mathrm{~m}, 3 \mathrm{H}, \mathrm{Ar}-\mathrm{H}), 4.2(\mathrm{~s}, 1 \mathrm{H}, \mathrm{N}-\mathrm{H}), \mathrm{MS}, \mathrm{m} / \mathrm{z}: 338.15(\mathrm{M}+)$; $\mathrm{C}_{21} \mathrm{H}_{16} \mathrm{ClN}_{3} \mathrm{~S}$ (Calcd. 393.89); Anal calcd. (\%) C, 58.75; H, 3.20; $\mathrm{N}$ 13.7; Found: C, 59.12; H, 3.62; N, 14.12.

\section{Compound III (3-amino-7-chloro-2-phenyl-quinazoline-4-(3H)- thione)}

Reddish brown crystalline solid (methanol); A mixture of 3-amino-7chloro-2-phenyquinazolin-4- $(3 \mathrm{H})$-one $(10 \mathrm{mmol}, 2.70 \mathrm{~g})$ and phosphorus penta sulphide (1 $\mathrm{mmol}, 2.43 \mathrm{~g}$ ) was reflux in anhydrous xylene $(100 \mathrm{ml})$ for $12 \mathrm{~h}$. The reaction mixture was filtered while hot, the solvent was evaporated and the residue was triturated with dimethyl sulphoxide $(10 \mathrm{ml})$ and filtered. The clear filtrate was poured into ice water, dried and recrystallised from ethanol to form reddish brown crystalline solid. (Yield: 65\%); m. p. 172-175 ${ }^{\circ} \mathrm{C}$; IR $\left(\mathrm{cm}^{-1}\right) v_{\max } \mathrm{Ar}-\mathrm{CH}_{\text {stretch }}\left(3262.75 \mathrm{~cm}^{-1}\right), \mathrm{C}-\mathrm{N}(1152.59$ $\left.\mathrm{cm}^{-1}\right), \mathrm{C}=\mathrm{N}\left(1664.49 \mathrm{~cm}^{-1}\right), \mathrm{C}=\mathrm{S}\left(1274.92 \mathrm{~cm}^{-1}\right), \mathrm{C}-\mathrm{Cl}\left(747.66 \mathrm{~cm}^{-1}\right), \mathrm{N}-$ $\mathrm{NH}$ (bend) (3343.75 cm-1), ${ }^{1} \mathrm{H}$ NMR (DMSO-d6, 400.13MHz), $\delta 7.24-$ $7.62(\mathrm{~m}, 5 \mathrm{H}, \mathrm{Ar}-\mathrm{H}), 7.23-7.41(\mathrm{~m}, 3 \mathrm{H}, \mathrm{Ar}-\mathrm{H}), 2.3(\mathrm{~s}, 1 \mathrm{H}, \mathrm{N}-\mathrm{H}) . \mathrm{MS}$, m/z: $288.12\left(\mathrm{M}+\right.$ ); $\mathrm{C}_{21} \mathrm{H}_{16} \mathrm{ClN}_{3} \mathrm{OS}$ (Calcd. 287.77); Anal calcd. (\%) C, 58.43; H, 3.5; N, 14.6; Found: C, 58.89; H, 3.82; N, 15.05 .

\section{Compound $\mathrm{III}_{\mathrm{i}}$ (1-(7-chloro-2-phenyl-4-thioxoquinazoline-3- (4H)-urea)}

White crystalline solid (methanol); A mixture of 1-(7-chloro-4-oxo2-phenylquinazoline-3 (4H-yl)) urea $(10 \mathrm{mmol}, 2.70 \mathrm{~g})$ and phosphorus penta sulphide ( $1 \mathrm{mmol}, 2.43 \mathrm{~g}$ ) was heated under reflux in anhydrous xylene $(100 \mathrm{ml})$ for $12 \mathrm{~h}$. The reaction mixture was filtered while hot, the solvent was evaporated and the residue was triturated with dimethyl sulphoxide $(10 \mathrm{ml})$ and filtered. The clear filtrate was poured into ice water, dried and recrystallised from ethanol to form white crystalline solid. (Yield: 64\%); m. p 162 $165^{\circ} \mathrm{C}$; IR $\left(\mathrm{cm}^{-1}\right) v_{\max } \mathrm{Ar}-\mathrm{CH}_{\text {stretch }}\left(3126.37 \mathrm{~cm}^{-1}\right), \mathrm{C}=\mathrm{S}\left(1243.35 \mathrm{~cm}^{-1}\right)$, $\mathrm{C}=\mathrm{N}\left(1598.62 \mathrm{~cm}^{-1}\right), \mathrm{C}-\mathrm{N}\left(1193.95 \mathrm{~cm}^{-1}\right) \mathrm{N}-\mathrm{NH}_{\text {stretch }}\left(3283.49 \mathrm{~cm}^{-1}\right)$, ${ }^{1} \mathrm{H}$ NMR (DMSO-d6, 400.13MHz), $\delta$ 7.24-7.63 (m, 5H, Ar-H), 7.24$7.42(\mathrm{~m}, 3 \mathrm{H}, \mathrm{Ar}-\mathrm{H}), 6.2$ (s, 1H, N-H). MS, m/z: $332.12(\mathrm{M}+)$; $\mathrm{C}_{15} \mathrm{H}_{11} \mathrm{ClN}_{4} \mathrm{OS}$ (Calcd. 330.79); Anal calcd. (\%)C, 54.46; H, 3.35; N, 16.94; Found: C, 54.86; H, 3.85; N, 17.32 .

Compound III $_{j}$ (1-(7-chloro-2-phenyl-4-thioxoquinazoline-3(4H)-thiourea)

White crystalline solid (methanol); A mixture of 1-(7-chloro-4-oxo2-phenylquinazoline-3 (4H-yl) $)$ thiourea $(10 \mathrm{mmol}, 2.70 \mathrm{~g})$ and phosphorus penta sulphide ( $1 \mathrm{mmol}, 2.43 \mathrm{~g})$ was heated under reflux in anhydrous xylene $(100 \mathrm{ml})$ for $12 \mathrm{~h}$. The reaction mixture was filtered while hot, the solvent was evaporated and the residue was triturated with dimethyl sulphoxide $(10 \mathrm{ml})$ and filtered. The clear filtrate was poured into ice water, dried and recrystallised from ethanol to form white crystalline solid. (Yield: 75\%); m. p. 163$165^{\circ} \mathrm{C}$; IR $\left(\mathrm{cm}^{-1}\right)$ vmax Ar-CH stretch $\left(3199.89 \mathrm{~cm}^{-1}\right), \mathrm{C}=\mathrm{S}(1266.56 \mathrm{~cm}-$ 1), $\mathrm{C}=\mathrm{N}\left(1671.85 \mathrm{~cm}^{-1}\right), \mathrm{N}-\mathrm{NH}_{\text {stretch }}\left(3027.51 \mathrm{~cm}^{-1}\right), \mathrm{C}-\mathrm{Cl}\left(692.51 \mathrm{~cm}^{-1}\right)$, ${ }^{1} \mathrm{H}$ NMR (DMSO-d6, 400.13MHz), $\delta$ 7.25-7.54 (m, 5H, Ar-H), 7.31$7.42(\mathrm{~m}, 3 \mathrm{H}, \mathrm{Ar}-\mathrm{H}), 2.3(\mathrm{~s}, 1 \mathrm{H}, \mathrm{N}-\mathrm{H}) . \mathrm{MS}, \mathrm{m} / \mathrm{z}: 345.12(\mathrm{M}+)$; $\mathrm{C}_{15} \mathrm{H}_{11} \mathrm{ClN}_{4} \mathrm{~S}_{2}$ (Calcd. 346.86); Anal calcd. (\%) C, 51.94; H, 3.2; N, 16.15; Found: C, 52.34; H, 3.46; N, 16.55 .

\section{Pharmacological activity}

The present biological study was approved by the Girijananda Chowdhury Institute of Pharmaceutical Science (GIPS) animal ethical committee (GIPS/IAEC/9). All the chemicals and solvents used for the pharmacological activity were purchased from SigmaAldrich. The newly synthesised compounds (IIa-IIIj) were tested for their antiepileptic and CNS depressant activities. The rota-rod test was performed to assess any probable changes in motor coordination induced by the test compounds. Seizure assay was carried out in accordance with the phase- 1 test of the anti-epileptic drug development (ADD) programme which was developed by the National Institute of Neurological and Communicative Disorders and Stroke [17].

\section{Anti-epileptic activity}

\section{Maximum electroshock seizure test}

\section{General procedure}

Albino mice of either sex, one to two weeks old weighing 20-25 g were used. The food was withdrawn 12-15 h before commencing the experiment while the food was withdrawn immediately before the experiment. The synthesised compounds were suspended in $30 \%$ of aqueous solution of polyethene glycol (PEG 400) and administered to the mice intraperitoneally in a standard volume of $0.5 \mathrm{ml}$ per $20 \mathrm{~g}$ body mass at a dose of $30 \mathrm{mg} \mathrm{kg}^{-1}, 100 \mathrm{mg} \mathrm{kg}^{-1}, 300 \mathrm{mg} \mathrm{kg}^{-1}$. Reference animals received $30 \%$ aqueous PEG 400 and 5, 5 diphenylimidazolidien-2, 4-dione (phenytoin) was used as a reference drug $\left(10 \mathrm{mg} \mathrm{kg}^{-1}\right)$. The maximum seizure was induced by application of an electrical stimulus $(50 \mathrm{~mA}$ at $60 \mathrm{~Hz})$ of $0.2 \mathrm{~s}$ in duration transmitted via corneal electrodes across the brain after 30 min and $4 \mathrm{~h}$ following drug administration. After applying the shock, the animals were observed for the type of convulsion produced, and the hind limb extensor response was taken at the endpoint [17-19].

\section{Neurotoxicity study}

\section{Rota-rod test}

\section{General procedure}

The rota-rod test was carried out in accordance with the method described in the literature [20]. The cardinal feature of the test is to ascertain the impairment of motor performance, ataxia, loss of skeletal muscle strength and acute neurotoxicity produced by drugs 
in preclinical studies. Albino mice weighing 20-24 g $(n=4-8)$, where $n$ represents the number of mice in a group, were trained to balance on the knurled wooden rotating rod $(0.032 \mathrm{~m}$ diameter $)$ that rotated at $6 \mathrm{~min}^{-1}$. Trained animals were treated with the test compounds at different dose levels (30 mg kg-1, $100 \mathrm{mg} \mathrm{kg}^{-1}, 300 \mathrm{mg} \mathrm{kg}^{-1}$ ) administered intraperitoneally. After $30 \mathrm{~min}$ and $4 \mathrm{~h}$, respectively the mice were placed onto the rotating rod for one minute. Neurological impairment was determined as the inability of the animal to remain on the rod for $1 \mathrm{~min}$.

\section{CNS depressant activity}

\section{Forced swimming test}

\section{General procedure}

The synthesised compounds were screened for their CNS depressant activity using Porsolt's behavioural despair (forced swimming) test. Male wister rats were placed in a chamber (diameter $0.45 \mathrm{~m}$, height $0.2 \mathrm{~m}$ ) containing water up to a height $0.15 \mathrm{~m}$ at $25 \pm 2{ }^{\circ} \mathrm{C}$. Two swim sessions were conducted an initial $15 \mathrm{~min}$ test, followed by a $5 \mathrm{~min}$ test session $24 \mathrm{~h}$ later. The animals were administered $(100 \mathrm{mg} / \mathrm{kg})$ the test compound i. p. $30 \mathrm{~min}$ before the test session. Then, the mice were dropped individually into the Plexiglas cylinder and left in the water for $5 \mathrm{~min}$. After the first $2 \mathrm{~min}$ of the initial vigorous struggling, the animals were immobile. A mouse was judged immobile if it floated in the water in an upright position and made only slight movements in order to prevent sinking. The period of immobility was accounted as passive floating without struggling and making only those movements which were necessary to keep its head above the surface of the water. Changes in the duration of immobilisation were evaluated using one-way analysis of variance (ANOVA) Dunnet's post hoc test9graphPad Instant Version 3.01 expressed as means \pm standard error of the mean (SEM). A $p$-value of less than 0.05 was considered statistically significant [21].

\section{RESULTS AND DISCUSSION}

\section{Physicochemical and spectral characterisation}

A novel series of 7-chloro-3-[substituted (amino/phenylamino)]-2phenyl quinazolin-4 $(3 \mathrm{H})$-one/thione derivatives and 1-(7-chloro-4oxo/-2-phenylquinazoline-3 (4H-yl)) substituted urea derivatives were synthesised as illustrated in the fig. 2 and characterised by using TLC, IR, ${ }^{1} \mathrm{H}-\mathrm{NMR}$ and mass spectroscopy. The synthesised compounds were soluble in methanol.

Table 1: the Preliminary anticonvulsant activity of compounds IIa-IIIj in mice (i. p.)

\begin{tabular}{|c|c|c|c|c|c|}
\hline \multirow[t]{2}{*}{ Compound } & \multirow[t]{2}{*}{ Dose (mg/Kg) } & \multicolumn{2}{|l|}{ MES $^{\mathbf{a}}$} & \multicolumn{2}{|c|}{ Toxicity $^{\mathbf{b}}$} \\
\hline & & $30 \mathrm{~min}$ & $4 h$ & $30 \mathrm{~min}$ & $4 h$ \\
\hline \multirow[t]{3}{*}{ IIa } & 30 & $0 / 1$ & $0 / 1$ & $0 / 4$ & $0 / 2$ \\
\hline & 100 & $3 / 3$ & $0 / 3$ & $0 / 8$ & $0 / 4$ \\
\hline & 300 & $1 / 1$ & $1 / 1$ & $0 / 4$ & $0 / 2$ \\
\hline \multirow[t]{3}{*}{ IIb } & 30 & $0 / 1$ & $0 / 1$ & $0 / 4$ & $0 / 2$ \\
\hline & 100 & $1 / 3$ & $0 / 3$ & $2 / 8$ & $0 / 4$ \\
\hline & 300 & $1 / 1$ & $0 / 1$ & $0 / 4$ & $0 / 2$ \\
\hline \multirow[t]{3}{*}{ IIC } & 30 & $1 / 1$ & $0 / 1$ & $0 / 4$ & $0 / 2$ \\
\hline & 100 & $2 / 3$ & $1 / 3$ & $2 / 8$ & $1 / 4$ \\
\hline & 300 & $1 / 1$ & $1 / 1$ & $3 / 4$ & $2 / 2$ \\
\hline \multirow[t]{3}{*}{ IId } & 30 & $0 / 1$ & $0 / 1$ & $0 / 4$ & $0 / 2$ \\
\hline & 100 & $2 / 3$ & $1 / 3$ & $0 / 8$ & $0 / 4$ \\
\hline & 300 & $1 / 1$ & $1 / 1$ & $0 / 4$ & $0 / 2$ \\
\hline \multirow[t]{3}{*}{ IIe } & 30 & $0 / 1$ & $0 / 1$ & $0 / 4$ & $0 / 2$ \\
\hline & 100 & $3 / 3$ & $1 / 3$ & $1 / 8$ & $0 / 4$ \\
\hline & 300 & $0 / 1$ & $1 / 1$ & $1 / 4$ & $0 / 2$ \\
\hline \multirow[t]{3}{*}{ IIf } & 30 & $0 / 1$ & $0 / 1$ & $0 / 4$ & $0 / 2$ \\
\hline & 100 & $3 / 3$ & $2 / 3$ & $0 / 8$ & $0 / 4$ \\
\hline & 300 & $1 / 1$ & $1 / 1$ & $0 / 4$ & $0 / 2$ \\
\hline \multirow[t]{4}{*}{ IIg } & 30 & $0 / 1$ & $0 / 1$ & $0 / 4$ & $0 / 2$ \\
\hline & 100 & $3 / 3$ & $1 / 3$ & $0 / 8$ & $0 / 4$ \\
\hline & 300 & $0 / 1$ & $0 / 1$ & $0 / 4$ & $0 / 2$ \\
\hline & 30 & $0 / 1$ & $0 / 1$ & $0 / 4$ & $0 / 2$ \\
\hline \multirow[t]{2}{*}{ IIh } & 100 & $2 / 3$ & $2 / 3$ & $0 / 8$ & $0 / 4$ \\
\hline & 300 & $1 / 1$ & $1 / 1$ & $0 / 4$ & $0 / 2$ \\
\hline \multirow[t]{3}{*}{ IIi } & 30 & $0 / 1$ & $0 / 1$ & $0 / 4$ & $0 / 2$ \\
\hline & 100 & $2 / 3$ & $2 / 3$ & $0 / 8$ & $0 / 4$ \\
\hline & 300 & $1 / 1$ & $1 / 1$ & $0 / 4$ & $0 / 2$ \\
\hline \multirow[t]{3}{*}{ IIj } & 30 & $0 / 1$ & $0 / 1$ & $0 / 4$ & $0 / 2$ \\
\hline & 100 & $3 / 3$ & $1 / 3$ & $0 / 8$ & $0 / 4$ \\
\hline & 300 & $0 / 1$ & $0 / 1$ & $0 / 4$ & $0 / 2$ \\
\hline \multirow[t]{3}{*}{ IIIa } & 30 & $0 / 1$ & $0 / 1$ & $0 / 4$ & $0 / 2$ \\
\hline & 100 & $1 / 3$ & $0 / 3$ & $1 / 8$ & $0 / 4$ \\
\hline & 300 & $1 / 1$ & $0 / 1$ & $1 / 4$ & $1 / 2$ \\
\hline \multirow[t]{3}{*}{ IIIb } & 30 & $0 / 1$ & $0 / 1$ & $0 / 4$ & $0 / 2$ \\
\hline & 100 & $2 / 3$ & $1 / 3$ & $0 / 8$ & $0 / 4$ \\
\hline & 300 & $0 / 1$ & $0 / 1$ & $0 / 4$ & $0 / 2$ \\
\hline \multirow[t]{3}{*}{ IIIC } & 30 & $1 / 1$ & $0 / 1$ & $0 / 4$ & $0 / 2$ \\
\hline & 100 & $1 / 3$ & $0 / 3$ & $0 / 8$ & $0 / 4$ \\
\hline & 300 & $0 / 1$ & $0 / 1$ & $3 / 4$ & $1 / 2$ \\
\hline \multirow[t]{3}{*}{ IIId } & 30 & $0 / 1$ & $0 / 1$ & $0 / 4$ & $0 / 2$ \\
\hline & 100 & $0 / 3$ & $0 / 3$ & $0 / 8$ & $0 / 4$ \\
\hline & 300 & $0 / 1$ & $0 / 1$ & $2 / 4$ & $0 / 2$ \\
\hline \multirow[t]{3}{*}{ IIIe } & 30 & $0 / 1$ & $0 / 1$ & $0 / 4$ & $0 / 2$ \\
\hline & 100 & $2 / 3$ & $1 / 3$ & $1 / 8$ & $0 / 4$ \\
\hline & 300 & $0 / 1$ & $0 / 1$ & $4 / 4$ & $0 / 2$ \\
\hline \multirow[t]{2}{*}{ IIIf } & 30 & $0 / 1$ & $0 / 1$ & $0 / 4$ & $0 / 2$ \\
\hline & 100 & $0 / 3$ & $0 / 3$ & $0 / 8$ & $0 / 4$ \\
\hline
\end{tabular}




\begin{tabular}{|c|c|c|c|c|c|}
\hline & 300 & $1 / 1$ & $1 / 1$ & $4 / 4$ & $0 / 2$ \\
\hline \multirow[t]{3}{*}{ IIIg } & 30 & $0 / 1$ & $0 / 1$ & $0 / 4$ & $0 / 2$ \\
\hline & 100 & $2 / 3$ & $1 / 3$ & $1 / 8$ & $0 / 4$ \\
\hline & 300 & $1 / 1$ & $1 / 1$ & $3 / 4$ & $0 / 2$ \\
\hline \multirow[t]{4}{*}{ IIIh } & 30 & $0 / 1$ & $0 / 1$ & $0 / 4$ & $0 / 2$ \\
\hline & 100 & $1 / 3$ & $2 / 3$ & $0 / 8$ & $0 / 4$ \\
\hline & 300 & $1 / 1$ & $1 / 1$ & $4 / 4$ & $0 / 2$ \\
\hline & 30 & $0 / 1$ & $0 / 1$ & $0 / 4$ & $0 / 2$ \\
\hline \multirow[t]{2}{*}{ IIIi } & 100 & $0 / 3$ & $0 / 3$ & $0 / 8$ & $0 / 4$ \\
\hline & 300 & $1 / 1$ & $0 / 1$ & $0 / 4$ & $0 / 2$ \\
\hline \multirow[t]{3}{*}{ IIIj } & 30 & $0 / 1$ & $0 / 1$ & $0 / 4$ & $0 / 2$ \\
\hline & 100 & $0 / 3$ & $0 / 3$ & $1 / 8$ & $0 / 4$ \\
\hline & 300 & $0 / 1$ & $0 / 1$ & $3 / 4$ & $0 / 2$ \\
\hline Phenytoin & 30 & $5 / 6$ & $5 / 6$ & --- & --- \\
\hline
\end{tabular}

MES: Maximum electroshock seizure, (a) Number of animal protected/number of animals tested, (b) Rota-rod test (number of animals exhibiting toxicity/number of animals tested)

The spectral data of compound I shows that $\mathrm{Ar}-\mathrm{CH}_{\text {stretch }}\left(3072 \mathrm{~cm}^{-1}\right)$, $\mathrm{C}=0\left(1751.33 \mathrm{~cm}^{-1}\right), \mathrm{C}=\mathrm{N}\left(1592 \mathrm{~cm}^{-1}\right)$, cyclic C-O-C stretch $(1060.47 \mathrm{~cm}$ $\left.{ }^{1}\right), \mathrm{C}-\mathrm{Cl}\left(680.72 \mathrm{~cm}^{-1}\right)$. Compounds Ila-IIj mainly characterised by the absorption width at the range $1752-1598 \mathrm{~cm}^{-1}$ which attribute the presence of a ketonic group $(\mathrm{C}=0)$ in the quinazoline moiety. Compounds IIa-IIIj showed the spectral range of 3323-3010 $\mathrm{cm}^{-1}$ which indicates the presence of $(\mathrm{C}-\mathrm{H})$ group in the aromatic ring. The spectral range of 3432-3027 $\mathrm{cm}^{-1}$ showed the presence of $\mathrm{N}-\mathrm{NH}$ stretching which also confirms the quinazoline skeleton in the respective compound. The presence of $\mathrm{C}$ - $\mathrm{Cl}$ group is deduced by the spectral range of 735-676 $\mathrm{cm}^{-1}$. The presence of $\mathrm{C}=\mathrm{S}$ is illustrated by the spectral range $1274-1233 \mathrm{~cm}^{-1}$. In addition, all the compounds displayed $\mathrm{C}_{4}-\mathrm{H}$ deformation. The mass spectra of the compounds were studied and the molecular ion peaks (M+), which were found consistent for all the compounds. The elemental analyses were within $\pm \%$ of the theoretical values. ${ }^{1} \mathrm{H}$ NMR spectra of IIa-IIIj exhibited different spectral ranges in which each appears as multiplet and triplet due to the presence of non-magnetically equivalent proton. The aromatic protons show at the peak at $\delta 6.5$ $8.725 \mathrm{ppm}$. The appearance of singlet protons around $\delta 3.46-4.5$ ppm for single protons in the ${ }^{1} \mathrm{H}$ NMR spectra might be assigned toNH-group. The appearance of singlet proton $\delta$ 2.34-2.51 ppm for three protons in its ${ }^{1} \mathrm{H}$ NMR spectra which might be assigned to aromatic methyl group confirms the formation of IIc/IIIc. The structures of the compounds are confirmed from the characteristics of the results obtained from analytical techniques.

\section{Pharmacological activity}

The anticonvulsant activity was evaluated by the MES test and the rota-rod test was used to evaluate neurotoxicity. CNS depressant activity was evaluated by forced swimming test.

\section{Anticonvulsant activity}

Out of all the compounds evaluated (table-1), IIb, IIc, IIe, IIIc and IIIe, exhibited anti-MES activity at either $100 \mathrm{mg} \mathrm{kg}^{-1}$ or $300 \mathrm{mg} \mathrm{kg}^{-1}$ in 30 $\mathrm{min}$; in addition, IIc and IIIc were also active at $30 \mathrm{mg} \mathrm{kg}^{-1}$. The compounds IIb, IIc, IIg, IIIb, IIIc, IIIg, were more active within $30 \mathrm{~min}$ than in $4 \mathrm{~h}$, indicating that they induced rapid onset of the action while III, IIIi, IIj, IIIj elicited late onset of the action.

Table 2: CNS depressant activity of compounds IIa-IIIj

\begin{tabular}{|c|c|c|c|}
\hline \multirow[t]{2}{*}{ Compound $^{\mathrm{a}}$} & \multirow{2}{*}{$\begin{array}{l}\text { Substitution } \\
\left(\mathrm{R} / \mathbf{R}_{1} / \mathrm{X}\right)\end{array}$} & \multicolumn{2}{|c|}{ Immobility time(s) } \\
\hline & & (mean \pm SEM) & Change from reference (\%) \\
\hline IIa & $\mathrm{H}$ & $175 \pm 11.34$ & 12.9 \\
\hline IIb & $2-\mathrm{Cl}$ & $180 \pm 10.23$ & 16. 12 \\
\hline IIc & $2-\mathrm{CH}_{3}$ & $236 \pm 10.45$ & 52. $25^{*}$ \\
\hline IId & $4-\mathrm{Cl}$ & $192 \pm 12.54$ & 23.87 \\
\hline IIe & $4-\mathrm{Br}$ & $198 \pm 17.56$ & 27.74 \\
\hline IIf & $4-\mathrm{NO}_{2}$ & $187 \pm 11.19$ & 20.64 \\
\hline IIg & $4-\mathrm{OCH}_{3}$ & $245 \pm 16.12$ & 58. $06^{*}$ \\
\hline IIh & $\mathrm{NH}_{2}$ & $192 \pm 15.28$ & 23. 87 \\
\hline IIi & 0 & $212 \pm 14.23$ & 36.77 \\
\hline IIj & $\mathrm{S}$ & $255 \pm 16.78$ & $64.51^{*}$ \\
\hline IIIa & $\mathrm{H}$ & $178 \pm 12.15$ & 14.83 \\
\hline IIIb & $2-\mathrm{Cl}$ & $195 \pm 13.18$ & 25.8 \\
\hline IIIc & $2-\mathrm{CH}_{3}$ & $261 \pm 12.35$ & $68.38^{*}$ \\
\hline IIId & $4-\mathrm{Cl}$ & $188 \pm 11.27$ & 21.29 \\
\hline IIIe & $4-\mathrm{Br}$ & $191 \pm 14.32$ & 23. 22 \\
\hline IIIf & $4-\mathrm{NO}_{2}$ & $182 \pm 15.75$ & 17. 41 \\
\hline IIIg & $4-\mathrm{OCH}_{3}$ & $254 \pm 17.85$ & $63.87^{*}$ \\
\hline IIIh & $\mathrm{NH}_{2}$ & $212 \pm 16.64$ & 36.78 \\
\hline IIIi & 0 & $210 \pm 18.92$ & 35.48 \\
\hline IIIj & $\mathrm{S}$ & $253 \pm 16.01$ & 63. $22^{*}$ \\
\hline PEG (control) & ---- & $155 \pm 10.54$ & ------ \\
\hline Carbamazepine ${ }^{b}$ & ------ & $260.62 \pm 15.24$ & 67.74 \\
\hline
\end{tabular}

(a) The compounds were tested at a dose of $100 \mathrm{mg} \mathrm{kg}^{-1}$ (i. p), (b) Tested at $30 \mathrm{mg} \mathrm{kg}^{-1}$ (i. p), ${ }^{*}$ Each value represents the mean \pm SEM of six rats (n=6) significantly different from the control at $p<0.05$ (Dunnet's test).

Mostly, the change in motor coordination was observed for IIb, IIc, IIg, IIi, IIId, IIIg, IIIi at the dose level of $300 \mathrm{mg} \mathrm{kg}^{-1}$. Compound IIc, IIg showed good anti-convulsant activity profile may be due to the presence of methyl/methoxy group presence of at $3^{\text {rd }}$ position of 
phenyl ring in quinazoline ring and compound IIi, IIIi, IIj, IIIj are having electro withdrawing group which probably increases the CNS activity as per the literature survey [13]. Compound IIj showed maximum protection at $30 \mathrm{~min}$ and $4 \mathrm{hr}$ which is having thiourea substitution in the ring probably show the anti-epileptic activity and similarly compound IIIg elicit increased in activity which may be due to the presence of methoxy group in the para position of phenyl ring which is attached to $3^{\text {rd }}$ position of quinazoline ring. Unlike Compound IIIg, Compound IIIh showed moderate protection at 30 min and $4 \mathrm{hr}$ in maximum electro shock seizure which is due to the presence of amine group at the $3^{\text {rd }}$ position of quinazoline ring. The amine group is an electron donor and increases the hydrogen bonding interaction with the target proteins or receptor for better CNS activity [22]. Finally, it may be concluded that IIc, IIg, IIj, IIIg, IIIh displayed better activity profiles compared with other derivatives as anti-convulsants with a sustained action.

\section{CNS depressant activity}

A few of the compounds tested (table 2, fig. 3) were noted as with potent CNS depressant properties, especially IIc, IIg, IIj, IIIc, IIIg and IIIj which exhibited remarkable activities revealing the highest duration of immobility comparable to $30 \mathrm{mg} / \mathrm{kg}$ of carbamazepine used as reference drug. Compound IIIc elicited excellent CNS depressant activity as compared to the control which may be due to the presence of methyl group in ortho position of phenyl ring which is attached to the $3^{\text {rd }}$ position of quinazoline scaffold. Similarly compound IIg, IIIg is having methoxy group in the phenyl ring. The CNS activity is probably due to the presence of these functional group as cited in the literature [13,23]. Similar case happened with the compoundIIj and IIIj where there is the presence of thiourea group which performed good CNS depressant activity [24]. The structural activity relationship based on the results observed indicated that the type of substituent attached to the $\mathrm{N}_{3}$ of the 7-chloro-(3-substituted phenylamino)-2-phenyl quinazoline-4 $(3 \mathrm{H})$-one scaffold modulated the activity. Attachment of the phenyl group alone does not elicit such favourable activity as compared with the substituted aryl nucleus. Electron withdrawing substituent decrease the activity in descending order, i.e., the higher the electronegativity the lower the activity, whereas the electron releasing substituent appear to be more favourable [25]. The bioisosterically related compounds IIIi, IIIj show similar and potent CNS depressant activity. Rest of the compounds showed mild to moderate CNS depressant activity.

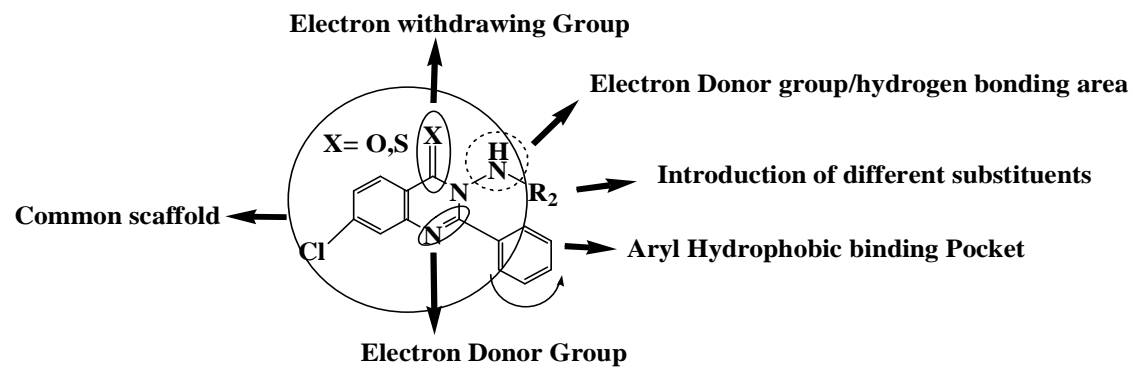

Fig. 1: Scaffold of the designed quinazoline derivatives

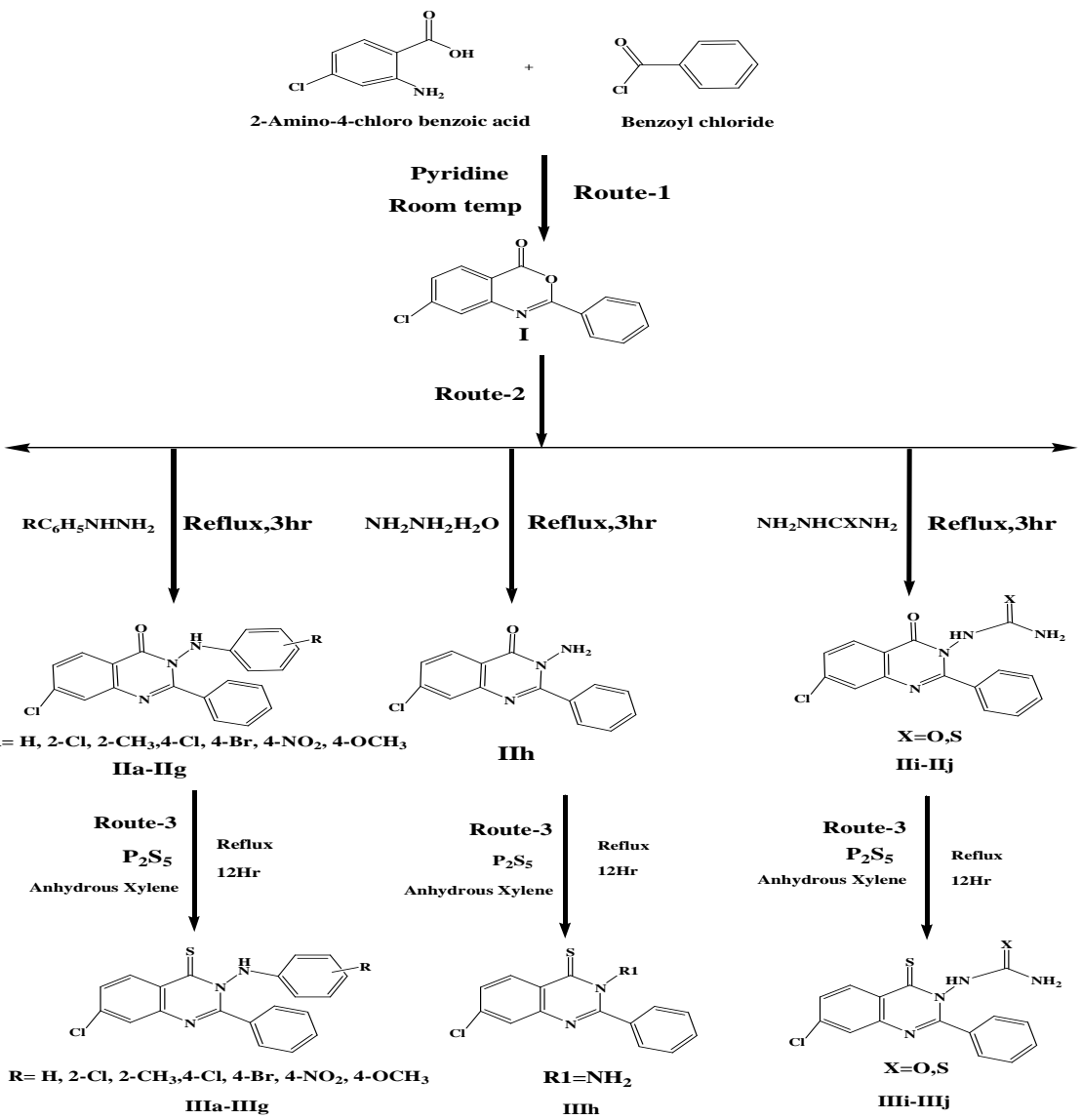

Fig. 2: Scheme for the synthesis of 7-chloro-3-[substituted (amino/phenyl amino)]-2-phenyl quinazolin-4 (3H)-one/thione derivatives and 1-(7-chloro-4-oxo/-2-phenylquinazoline-3 (4H-yl)) substituted urea 


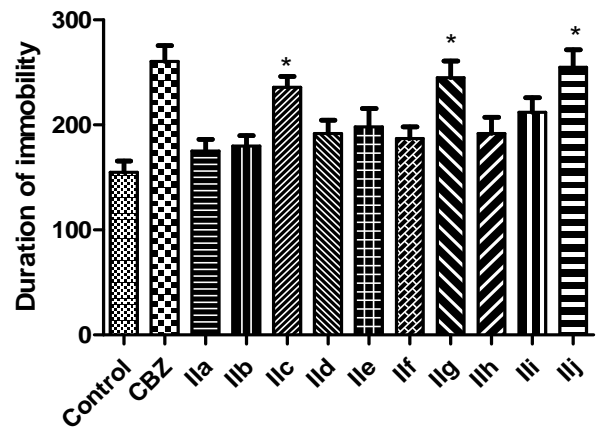

Tested compounds

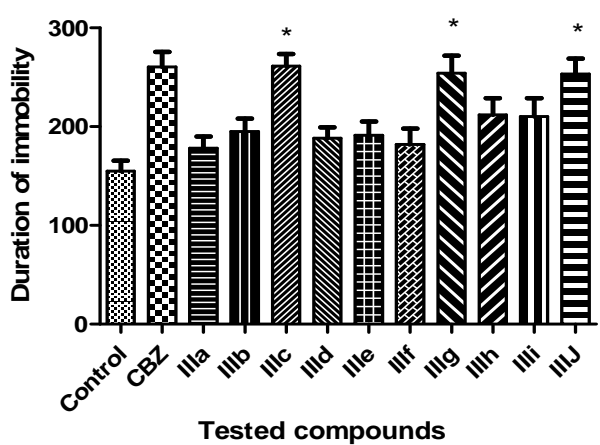

Fig. 3: Duration of immobility by porsolt's behavioural despair test, ${ }^{*}$ Each value represents the mean \pm SEM of six rats $(n=6)$ significantly different from the control at $p<0.05$ (Dunnet's test); CBZ: Carbamazepine

\section{CONCLUSION}

A series of quinazoline derivatives with a common skeleton were synthesised by replacing different substituted phenyl hydrazine/ urea/thiourea derivatives at $3^{\text {rd }}$ position of quinazoline pharmacophore by suitable techniques. The quinazoline derivatives obtained from this research work indicates that the methyl/methoxy group in phenyl hydrazine ring at $3^{\text {rd }}$ position, amine, thiourea substitution at $3^{\text {rd }}$ position of quinazoline derivatives are essential for anti-convulsant and CNS depressant activity. Compounds IIc, IIg, IIj, IIIc, IIIg, IIIj, IIIh were found to be a potent compound which may be effective as a potential source for the development of CNS depressant and an anticonvulsant compound having common quinazoline scaffold with lesser side effects. A further study is going on for in vitro study of the newly synthesised and pharmacologically potent quinazoline molecules.

\section{ACKNOWLEDGMENT}

The authors acknowledged the assistance of SAIF, North-Eastern Hill University, Shilong, Meghalaya for analytical support regarding spectral analysis.

\section{CONFLICT OF INTERESTS}

Declared none

\section{REFERENCES}

1. Donner EJ, Snead OC. New generation anticonvulsants for the treatment of epilepsy in children. J Am Soc Exp Neuro Ther 2006;3:170-80.

2. Mahendra RS, Mangesh G, Kailash GB, Shashikant VB, Ana N Kalyan CA, Nisheeth CD, et al. Synthesis and anticonvulsant activity of clubbed thiazolidinone-barbituric acid and thiazolidinone-triazole derivatives. ARKIVOC 2007;XIV:58-74.

3. Mochizucki D. Serotonin and noradrenaline reuptake inhibitors in animal models of pain. Human Psychopharmacol 2004;19:S15S19.
4. Brodie MJ. Established anticonvulsants and treatment of refractory epilepsy. Lancet 1990;336:350-4.

5. Schmidt D, Löscher W. Drug resistance in epilepsy: putative neurobiologic and clinical mechanisms. Epilepsia 2005;46:858-77.

6. Kwan P, Brodie MJ. Early identification of refractory epilepsy. New Engl J Med 2000;342:314-9.

7. Pollard JR, French J. Antiepileptic drugs in development. Lancet Neurol 2006;5:1064-7.

8. Dichter MA, Brodie MJ. New antiepileptic drugs. New Engl J Med 1996;334:1583-90.

9. Brodie MJ, Dichter MA. Antiepileptic drugs. New Engl J Med 1996;334:168-75.

10. Kwan P, Brodie MJ. Drug treatment of epilepsy: when does it fail and how to optimize its use? CNS Spectr 2004;9:110-9.

11. Siddqui N, Akhtar JM, Ali R, Azad B. An updated review: emerging anticonvulsants. Int J Pharm Biosci Arch 2010;1:404-15.

12. Dimmock JR, Pandeya SN, Quail JW, Pugazhenthi U, Allen TM, Kao GY, et al. Evaluation of the semicarbazones, thiosemicarbazones and bis-carbohydrazones of some aryl alicyclic ketones for anticonvulsant and other biological activities. Eur J Med Chem 1995;30:303-14.

13. Mahato AK, Srivastava B, Nithya SC. Chemistry, structure activity relationship and biological activity of quinazolin-4 (3H)-one derivatives. Inventi Rapid Med Chem 2011;2:400-2.

14. Ilangovan P, Ganguly S, Pandit V. Design and synthesis of novel quinazolinone derivatives as broad spectrum anticonvulsant and antimicrobial agent. J Pharm Res 2010;3:703-6.

15. Hemlata K, Girija K. Synthesis of some novel 2, 3disubstituted quinazolinone derivatives as analgesic and anti-inflammatory agents. Int J Pharm Pharm Sci 2011;3:103-6.

16. Adel S, Azab E, Mohamed A, Omar A, Alaa AM, Naglaa I, et al. Design, synthesis and biological evaluation of novel quinazoline derivatives as potential antitumor agents: molecular docking study. Eur J Med Chem 2010;45:4188-98.

17. Krall RL, Penry JK, White BG, Kupeferberg HJ, Swinyard EA. Antiepileptic drug develpment: II. Anticonvulsant drug screening. Epilepsia 1978;19:409-28.

18. Porter RJ, Cereghino JJ, Gladding GD, Hessie BJ, Kupferberg HJ, Scoville B, et al. Antiepileptic drug development program. Cleveland Clin Quart 1984;51:293-305.

19. Ladda PL. Screening of Riccinus communis Linn. Leaves for anticonvulsant and analgesic activity. Asian J Pharm Clin Res 2014;7:110-4.

20. Rajak H, Desmukh R, Aggarwal N, Kashaw S, kharya MD, Mishra $\mathrm{P}$, et al. Synthesis of novel 2, 5-disubstituted 1, 3, 4-thiadiazoles for their potential anticonvulsant activity: pharmacophoric model studies. Arch Pharm 2009;342:453.

21. Porsolt RD, Anton G, Blanet N, Jalbre M. Behavioral despair in rats: a new model sensitive to antidepressant treatments. Eur J Pharmacol 1978;47:379-91.

22. Ibrahim MK, El-Adl K, Al-Karmalawy AA. Design, synthesis, molecular docking and anticonvulsant evaluation of novel 6iodo-2-phenyl-3-substituted-quinazoli-4(3H)-ones. Bull Faculty Pharm Cairo University 2015;53:101-16.

23. Adel S, Kamal EH. Design and synthesis of novel 7-amino Quinazoline derivatives: antitumor and anticonvulsant activities. Bioorg Med Chem Lett 2012;22:1879-85.

24. Kashaw SK, Kashaw V, Mishra P, Jain NK. Design, synthesis and potential CNS activity of some novel 1-(4-substituted-phenyl)3-(4-oxo-2-propyl-4H-quinazolin-3-yl)-urea. ARKIVOC 2008; XIV:17-26.

25. Kashaw SK, Kashaw V, Mishra P, Jain NK, Stable JP. Synthesis, anticonvulsant and CNS depressant activity of some new bioactive 1-(4-substituted-phenyl)-3-(4-oxo-2-phenyl/ethyl4H-quinazolin-3-yl)-urea. Eur J Med Chem 2009;44:4335-43.

\section{How to cite this article}

- Biswajit Dash, Suvakanta Dash, Damiki Laloo. Design and synthesis of 4-substituted quinazoline derivatives for their anticonvulsant AND CNS depressant activities. Int J Pharm Pharm Sci 2017;9(1):165-172. 